\title{
HIDROLOGIA COMPARATIVA DE LAS BOCAS DE DOS ANTIESTUARIOS DE BAJA CALIFORNIA
}

por:

\author{
SAUL ALVAREZ BORREGO \\ MANUEL DE JESUS ACOSTA RUIZ \\ JOSE RUBEN LARA LARA \\ Centro de Investigación Científica y \\ Educación Superior de Ensenada, B.C. \\ Espinoza 843, Ensenada, B.C. \\ (Recibido: junio 20, 1977) \\ Ciencias Marinas Vol. 4 Núm 11977.
}

\section{RESUMEN}

Durante la primavera y verano de 1975, se llevaron a cabo cuatro muestreos en las bocas de Bahía San Quintín y el Estero de Punta Banda. El objetivo fue estudiar la variación de temperatura, salinidad oxígeno disuelto, pH y algunas variables meteorológicas, en función del tiempo en ciclos diurnos. El. estudiàr ambas lagunas costeras nos permiltirá hacer comparaciones para encontrar diferencias y caracte5.sticas comuries que puedar ser extrapoladas a otros anticstuarios. También se presentan resultados preLiminares de otoño en San Quintín.

ABSTRACT

During spritg and summer of 1975 four samplings were carred in two at the mow of San Quintin Bay and two at the neth of Estero de Punta Banda, with the oojectiy to study the variation of temperature, salirity, disobved oxyuen, $p H$ and some meteorological varlables as fuctions of time, in diuna cycies. This is part of a study that intends to give an inrrastructure for the development of maricuiture in the antiestuaries of Baja California. To study San Quiritin Bay and the Estero de Punta Banda will allow us to make comparisons with the objective to find common characteristics that can be extrapolated to other antiestuaries. Also, in this report we prom sent-preliminary results from a sampling made in fall. 
INTHODUCCION

La hidrología de Bahía San Quintín y el [stero de Punta 3anda ha sido estudiada con el objetivo primordial de proporcionar una infraestructura cientifica para el desarrollo de maricultivos (Acosta Ruíz y Alvarez Borrego, 1975; Lara Lara y Alvarez Borrego, 1975; Alvarez Borrego, Ballesteros Grijalva y Chee Barragán, 1975; y Alvarez Borrego y Chee Barragán, 1976). El énfasis hasta ahora había sido el determinar la distribución espacial de diversos parámetros ecológicos físico-químicos y su cambio en función del tiempo a lo largo de ciclos anuales; además de estudiar los parámetros relacionados con la productividad orgánica primaria. Alvarcz Borrego y López Alvarez (1975) presentaron algunos resultados sobre la determinación de biomasa por grupos taxonómicos de fitoplancton. Para un mejor cntendimiento de los procesos que afectarán a cualquier grupo de especies que se cultiven en lagunas costeras semejantes a Bahía San Quintín y al Estero de Punta Banda, es necesario tener un mejor conocimiento de la dinámica de los mismos, el campo de la velocidad de corrientes y su compleja fluctuación en función del tiempo. Este campo dependerá de las mareas, la geometría de la laguna costera, especialmente su batimetría, los vientos y los efectos termohalinos de incremento de la temperatura y evaporación. Del campo de la velocidad depende la renovación del agua donde se esté realizando el cultivo, y por lo tanto el suplemento de

alimento, sobre todo en el caso de cultivos de macro-algas $y$ de moluscos filtro-alimentadores.

Redfield, Ketchum y Richards (1963) indicaron el poco conocimiento que se tienc sobre la hidrología de sistemas antiestuarinos, comparido con los ampl iamente estudiados sistemas estuarinos. Las especulaciones que se han hecto sobre los sistemas antiestuarinos, en que la evaporación es mayor que cualquier aporte de agua dulce, se han basado en el conocimiento del mar Mediterráneo. Por ejemplo se especulaba que el contenído de nutrientes tendería a disminuir en el sistema antiestuarino cor respecto al océano abierto adyacente, debido a una circulación termohalina que produciría en la boca una corriente en el fondo hacia afuera, de agua con mayor salinidad; y en la superficie, una corriente hacia adentro de agua con menor salinidad. Alvarez Borrego y Chee Barragán (1976) denostraron que a lo largo de todo el año la concentración de nutrientes en Bahía San Quintín aumenta de la boca hacia el interior, es decir, es mayor en el interior de la Bahía que en el océano abierto adyacente. 
El objetivo del presente trabajo es el determinar la hidrología de las bocas de dos sistemas antiestuarinos en Baja California, Bahía San Quintín y el Estero de Punta Banda, y su cambio estacional a través de un año. Esto nos podra permitir en el futuro hacer cálculos de flujo y balances de materiales, sobre todo de fitoplancton y de nutrientes en solución. Al estudiar simultáneamente dos sistemas antiestuarinos podemos realizar comparaciones que quizás nos permitan establecer características lo suficientemente comunes para ser extrapoladas a cualquier otro sistema antiestuarino.

En este trabajo se presentan de una manera preliminar, los resultados obtenidos de los muestreos de primavera y verano de 1975; incluyendo salinidad, temperatura, oxígeno disuelto, pH y condiciones meteorológicas. Además, se presentan resultados preliminares obtenidos en otoño en San Quintín.

El Estero de Punta Banda es una laguna costera localizada a lo largo de la orilla sureste de la Bahía de Todos Santos (Acosta Ruíz y Alvarez Borrego, 1974). Su boca se encuentra aproximadamente a 8 millas náuticas del mar abierto. Fuera de la Bahía de Todos Santos, al suroeste de Punta Banda se encuentra una zona de surgencias (Chávez de Ochoa, 1975). La boca del Estero no es mayor de $150 \mathrm{~m}$ en mareas bajas.

Bahía San Quintín se localiza a $200 \mathrm{~km}$ al sur de Ensenada, Baja California Norte. En el mar abierto inmediatamente al sur de la entrada de la Bahía ocurren surgencias intensas (Dawson, 195i). La boca tiene un poco más de un kilómetro de ancho (ver Chávez de Nishikawa y Alvarez Borrego, 1974).

\section{MATERIALES Y METODOS}

Se realizaron dos muestreos en cada boca en mayo y agosto, para estudiar las condiciones de primavera y verano. En caia muestreo se anclaba la embarcación "SIRIUS I" de 35 piés de eslora, propiedac del CICESE, a manera de plataforma fija. Se tondzon muestras cada hora por períodos de 26 horas trabando incluir un ciclo de radiación solar y de mareas. ademas, en primavera se muestrearon 4 estaciones hacia el interion del Estero de Punta Banda, $y$ en verano se muestrearon astaciones hacia i interior de ambos lugares ( $F i g . j$ ). Er Sar ouintín se miestreo el 7 y 8 de nayo, y el 14 y 15 de ugosto; y en el Estero de Punta Banda el 26 y 27 de mayo, y el 27 y 28 de agosto. El muestreo de otoño, se realizó el 26 y 27 de noviembre. 
Distribución espacial de:

$T^{\circ} \mathrm{C}$

$5 \%$

$\mathrm{O}_{2} \mathrm{ml} / \mathrm{it}$

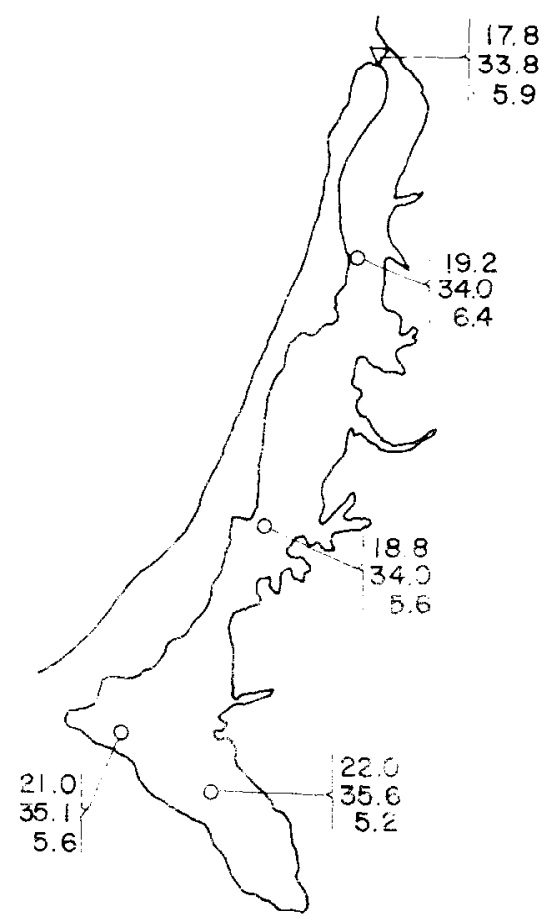

ESTERO PUNTA BANDA

PRIMAVERA

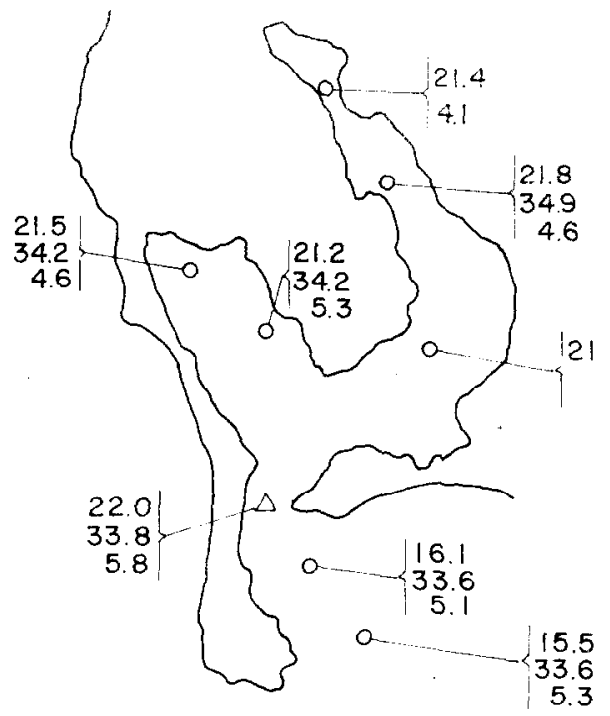

BAHIA SAN QUINTIN VERANO

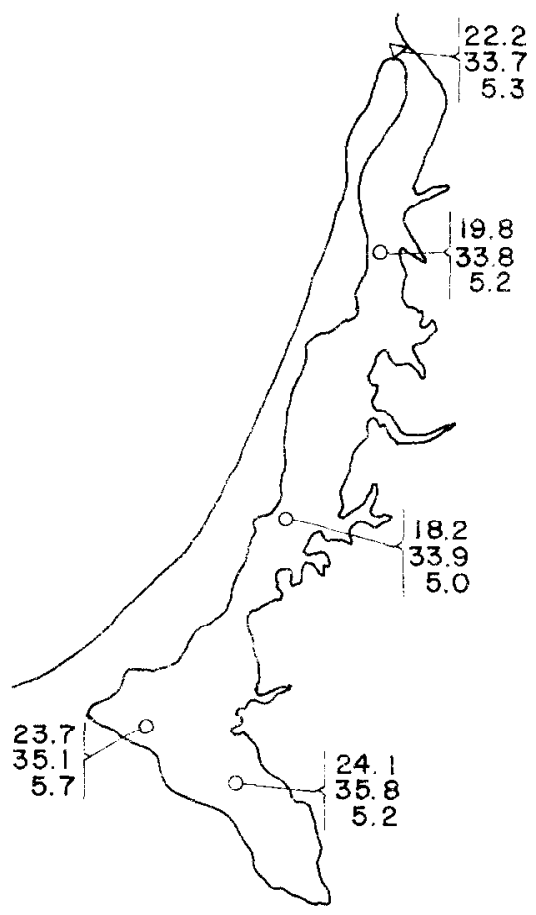

ESTERO PUNTA BANDA VERANO

Fig. 1. - Localización de las estaciones de muestreo en Bahía Sar Quintín y en el Estero de Punta Banda, mostrando la distribución espacial de temperatura salinidad y oxígeno disuelto. 
Se colectaron muestras de agua de superficie y de fondo para determinaciones de temperatura, salinidad y oxígeno disuelto. $Y$ solamente de superficie para $\mathrm{pH}$. No fue posible tomar datos de $\mathrm{pH}$ en verano debido a fallas en el potenciómetro. En el muestreo de noviembre se determinó la dirección e intensidad de la corriente en la superficie y aproximadamentc a un metro del fondo. Además, se tomaron datos de temperatura ambiental y dirección e intensidad del viento para los propósitos del presente trabajo.

La salinidad se determinó mediante un salinómetro Beckman, modelo 118 WA200; la temperatura mediante termómetros reversibles y termómetros de cubeta; el oxígeno por el método macroWinkler descrito por Strickland y Parsons (1965); el pH utilizando electrodos de vidrio y un potenciómetro Orion, de baterias, modelo 401, y las corrientes mediante un correntómetro tipo Savonius.

La colecta de agua de fondo se hizo con botellas Nansen recubiertas con teflón, y la de superficie con botellas Van-Dorn. Antes de realizar cada muestreo se leía la profundidad mediante una ecosonda y en base a esto se situaba la botella de fondo aproximadamente a 1 metro del mismo. La profundidad media en la boca de San Quintín es de cerca de 9 metros y en el Estero de Punta Banda 7 metros.

La intensidad y dirección del viento se midió con un anemómetro Kahlsico modelo 03 AM 120. La salinidad se determinó en el laboratorio, mientras que el resto de las propiedades se determinaron en el campo. Las gráficas de mareas se construyeron cori datos del calendario publicado por la Secretaría de Marina, con su corrección por tiempo y espacio para el lugar estudiado.

RESULTADOS

En general la variación de temperatura y salinidad a través de un ciclo diurno tuvo tendencias más claramente marcadas en Bahía San Quintín que en el Estero de Punta Banda (Fig. 2). Especialmente notable es la alta correlación entre estas variables ocurrida en primavera en Banía San Quintín. En el Estero de Punta Banda se aprecian cambios bruscos de los valores, mientras que en Bahía San Quintín en general la variación fue suave. En Bahia San Quintín, en verano, alrededor de las trece horas se detectó un fenómeno más claramente mostrado por la temperatura pero corroborado por la salinidad ( $F$ ig. 2), consistente en una anomalía que aumentó los valores de ambas propiedades con 

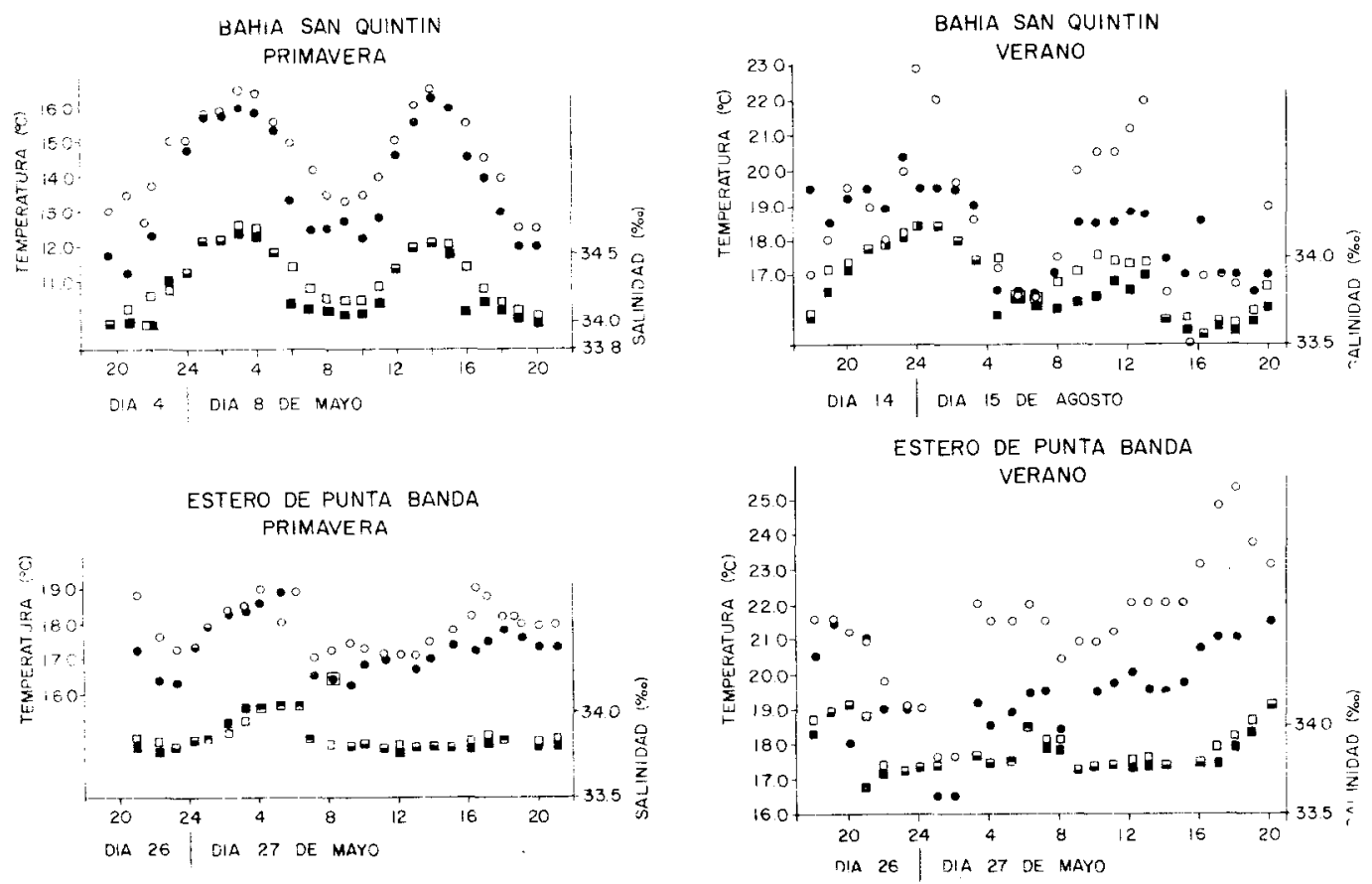

Fig. 2.- Variación de temperatura y salinidad en las bocas de San Quintín y el Estero. Círculos y cuadros claros representan valores de superficie y los y los oscuros de fondo. Los círculos representan temperatura.

respecto a la tendencia general de variación. El cambio brusco más marcado en el Estero de Punta Banda ocurrió a las seis horas en primavera. En general la temperatura fue mayor en la superficie que en el fondo, con diferencias hasta de más de $3^{\circ} \mathrm{C}$ en San Quintín en verano, $y$ hasta más de $4^{\circ} \mathrm{C}$ en el Estero. Las diferencias de los valores de salinidad, de superficie y de fondo no son tan notables como las de temperatura.

Con relación a la salinidad, la columna de agua fue menos homogénea en San Quintín que en el Estero. La salinidad fue en general igual o mayor en la superficie que en el fondo. En verano en Bahía San Quintín se detectó una inversión en el gradiente de temperatura, en la colunna de agua, de las 15:00 a jas 23:00 horas del primer día; a las 03:00 horas; de las 06:00 a las 07:00 horas y de las 14:00 a las 18:00 horas del segundo día, con tem. peraturas mayores en el fondo que en la superficie (Fig. 2). Estc implica que hubo una inversión en el gradiente de densidad. Para corroborar esto, se tuvo especial cuidado en las mediciones de temperatura realizadas en otoño en San Quintín (Fig. 3); en efecto, una vez 

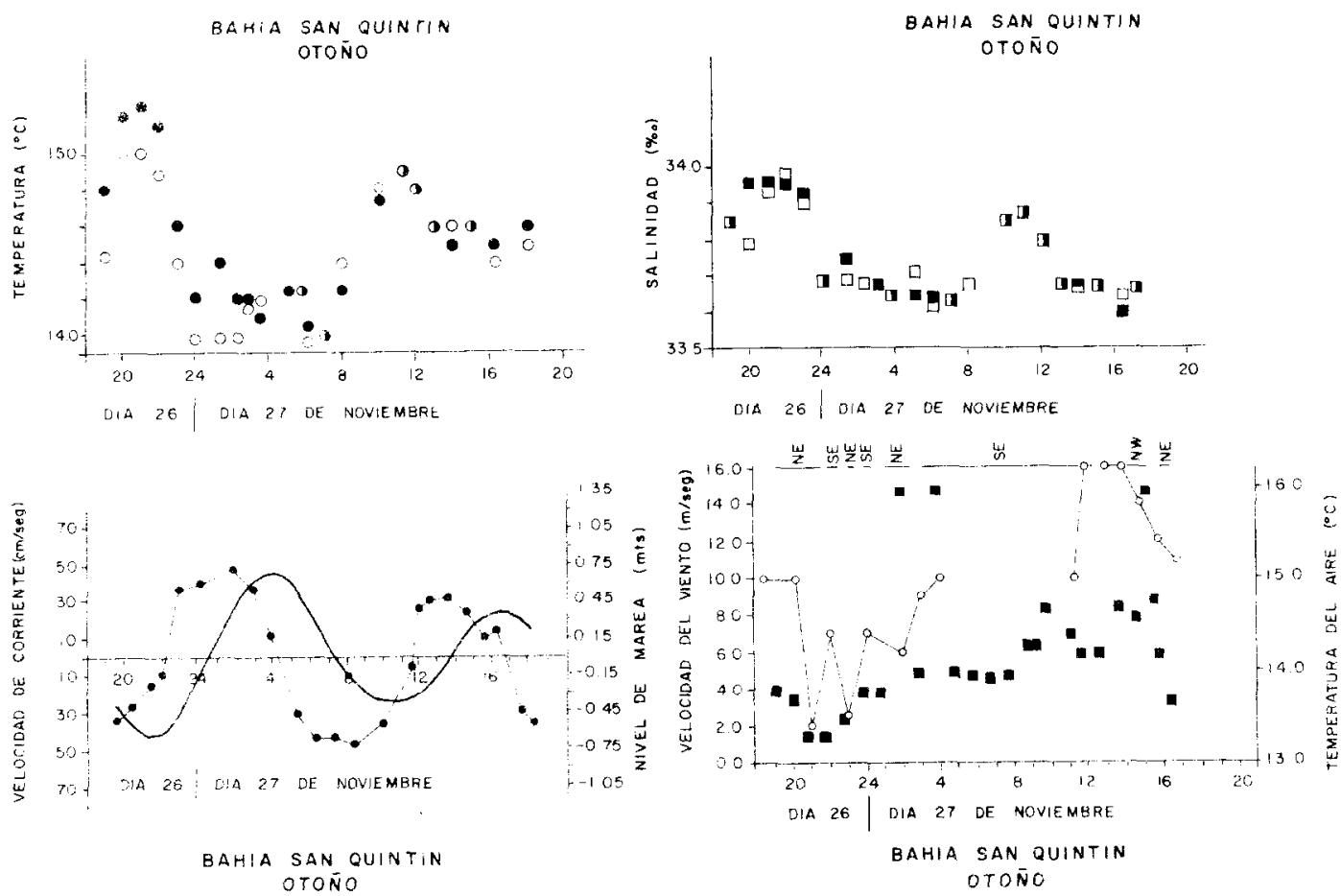

Fiq. 3.- Variación de temperatura, salinidad, velocidad de córrientes, marea, velocidad del viento y temperatura del aire en la boca de San Quintír en el muestreo de otorio. Círculos claros representar valores de superficie y los oscuros de fondo, en las greficas de $T^{\circ} \mathrm{C}$ y $5 \% / 00$. Ld linea continua es la grafica de marea. Círculos claros represen$\tan l a$ velocilad del viento.

más se detectaron temperaruras en el fondo un poco mayores que en la superficicie. Aunque no presentamos las gráricas de sigma-t, los resultados indican que en algunas ocasiones las fuertes corricntes de marea provocan temporalmente el que se encuentre agua en la superficie de mayor densidad que en el fondo.

Contrario a 1 a salinidad y temperatura, la variación de oxigeno disuelto fue más irregular en San Quintín que en e. Estero (Fig. 4). [1 phl fue casi invariable en el Estero de Punta Banda, mientras que en San Quintín su variacion fue claramente marcada, con una alta correlación con la sainidad, temperatura y oxígeno disueito (Fig. 2 y 4).

Los vientos fueron más intensos en San Quintín que en el Estero de Punta banda, tanto en primavera como en verano (íig. 5). En el Estero se registraron algunos períodos de calma, mientras que en San Quintín la velocidad mínima del viento fue $4.0 \mathrm{~m} / \mathrm{seg}$. En San Quintín se registraron vientos 

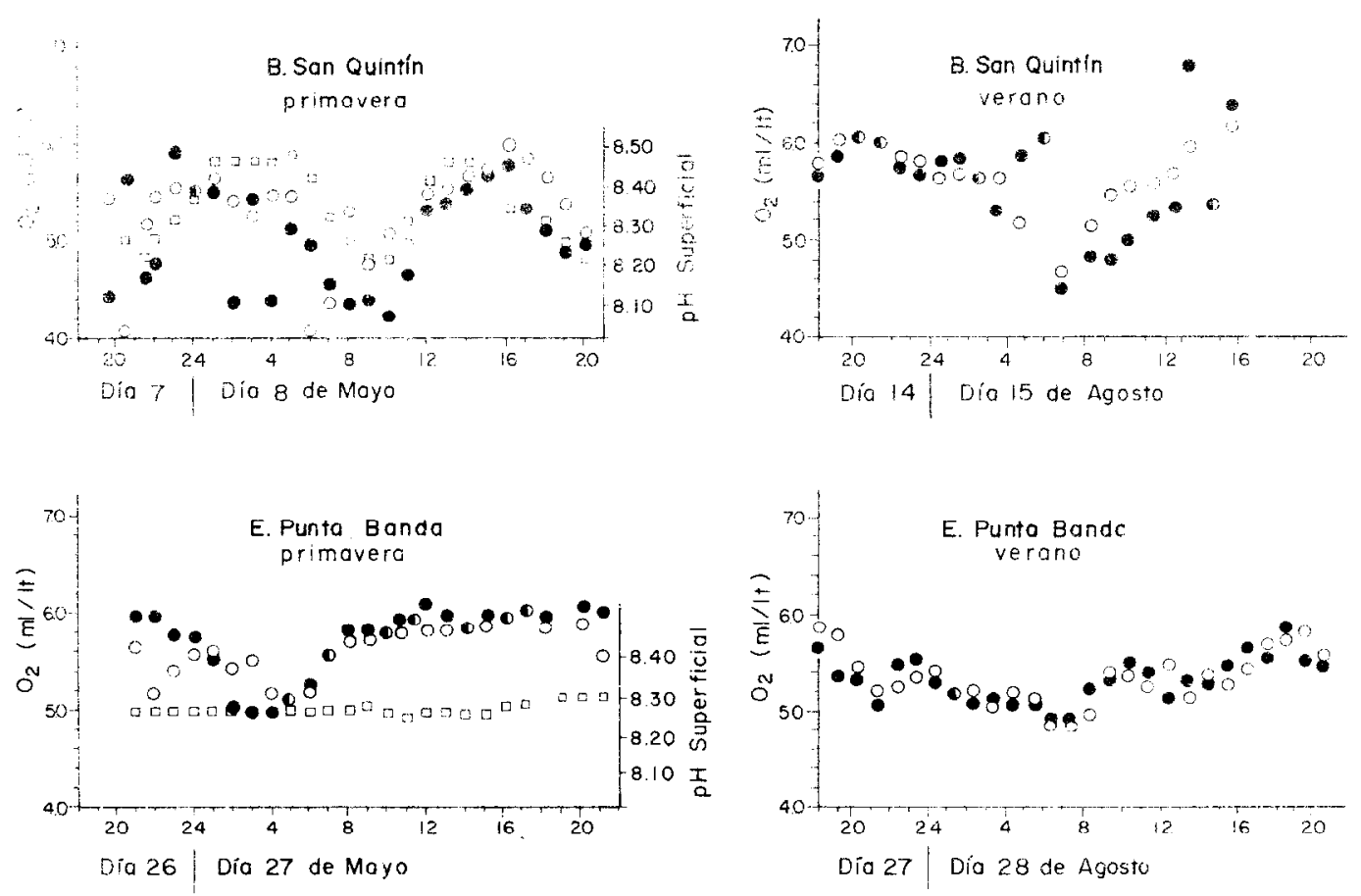

Fig. 4.- Variación de oxígeno disuelto y pH en las bocas de San Quintín y el Estero. Círculos y cuadros claros representan valores de superficie y los oscuros de fondo. Los cuadros representan valores

con componente del oeste persistente casi durante las veinticuatro horas del día, mientras que en el Estero se presentó el ciclo de brisas de mar y tierra. La temperatura del aire fue más baja y más estable en San Quintín que en el Estero.

La velocidad de corriente fue medida de una manera apropiada por primera véz en el muestreo de otoño en Bahía San Quintin ( $\mathrm{Fig} .3$ ). La velocidad máxima registrada fue $55 \mathrm{~cm} / \mathrm{seg}$ en flujo. La gráfica muestra la velocidad promedio en la columna de agua, aunque en general se registraron velocidades iggeramente mayores en la superficie.

\section{DISCUSIONES}

Resultados obtenidos anteriormente han mostrado que la salinidad y temperatura generalmente tienden a aumentar de la boca al interior de estos dos antiestuarios (Acosta fuiz y Alvarez Borrego, 1974, y Chávez do Nishikawa y Alvarez Borrego, 1974). De acuerdo con esto, al estar midiendo estas variables er las bocas, en función del tiempo, debe haber. una correlación estrecha con la marea, con la salinidad y temperatura aumentando en reflujo $y$ iceversa. En efecto, en el muestreo de primavera en San Quintín se detectó una 

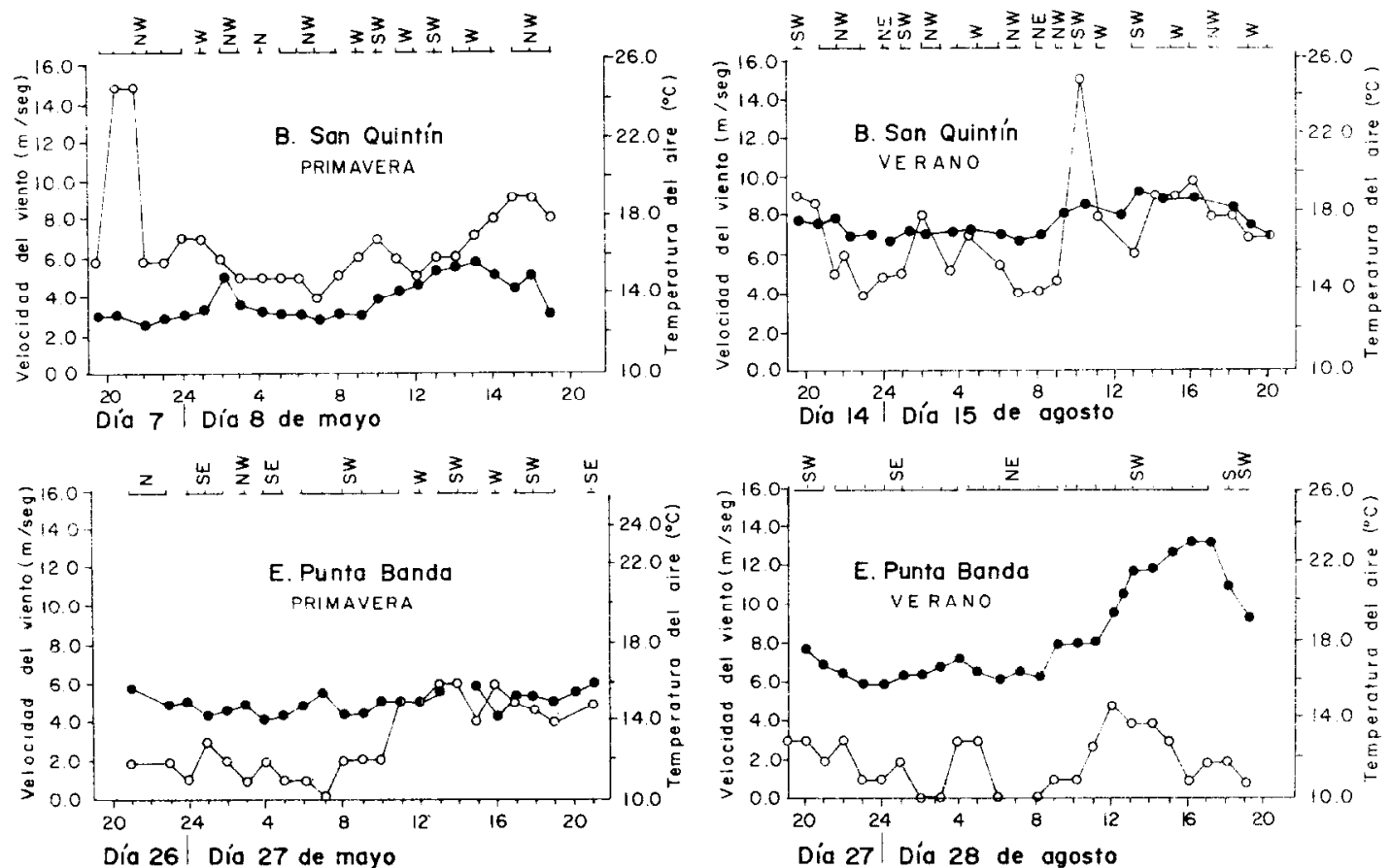

F1g. 5.- Variación de la velucidad del viento y la temperatura del aire en San Quintín y el Estero. Círculos claros representan la velocidad del viento y los oscuros lá temperatura.

correlación casi perfecta entre la temperatura, la salinidad la marea, y también el oxígeno y el pH (Fig. 2 y 4). Sin embargo, en los demás casos no es evidente este tipo de correlación. Existen diversos factores que pueden causar variaciones irregulares de estas propiedades. v.g.: calentamiento y evaporación no uniforme en el interior de los antiestuarios debido a ura batimetría irregular, con canales y bajos; 1 a presencia de corrientes a lo largo de la playa en el extrior de las bocas; las condiciones cambiantes en la zona oceánica adyacente a las bocas como los cambios producidos por una surgencia; y con relación a la concentración de oxigeno, el oleaje variable de acuerdo con las condiciones de los vientos. Cabrera Muro (1972) y Contreras Rivas (1973) detectaron una corriente paralela a la playa frente a la boca del Estero de Punta Banda, con direrección norte. Los cambios bruscos de temperatura y salinidad detectados en algunas ocasiones en la boca del Estero, Lales como el ocurrido a las 6 de la mañana en primavera (Fig. 2), son seguramente debido a este tipo de corriente paralela a la playa, que provoca el desplazamiento del agua que sale del Estero, de tal marera que al comenzar a subir la marea entra agua "nueva" no mezclada, y se registra en la boca una súbita disminución de tempcratura y salinidad. Algunas de las anomalías registradas en San Quin. tin ( $i g$. 2) fueden deberse también a este tipn de corrien tes a 10 iargo de la playd. 
El intenso oleaje que comúnmente ocurre frente a la estrecha boca del Estero, aunado a las fuertes corrientes de marea (más fuertes en el. Estero que en San Quintín, por lo estrecho de la boca del primero), son causantes de la mayor homogeneidad de la columna de agua en el Estero. En general, en ambos lugares, la columna de agua es más homogénea durante el reflujo.

Seguramente la presencia de surgencias en la zona oceánica adyacente a la boca de San Quintín son la causa de que se hayan registrado temperaturas más bajas que en el Estero, en el agua y en el aire. Vientos persistentes del noroeste registrados en San Quintín ( $F i g .4)$ pueden haber causado estas surgencias.

La experiencia adquirida con este trabajo preliminar nos enseña que difícilmente se pueden obtener conclusiones fuertes con series de tiempo tan pequeñas. Por lo cual, futuros muestreos deben abarcar períodos del orden de unas dos o tres semanas, en lugar de un día.

\section{RECONOCIMIENTOS}

Agradecemos al Sr. Antonio Hovelo el habernos permitido utilizar sus instalaciones en el Estero de Punta Banda. Agradecemos a los compañeros Gilberto Gaxiola Castro, Sila Najera Martínez, Bernardo P. Flores Baez, Felipe Salinas, Artemio Gallegos, Salvador Galindo Bect, Talpa Lara, Lucía Vargas, Claudia Valdéz, Enrique Parra, Refugio Conzález, Luis A. Galindo Bect y Marcos Miranda Aguilar, su valiosa colaboración en los trabajos realizados.

\section{BIBLIOCRAFIA}

Acosta Ruiz, M. de J. y S. Alvarez Borrego. 1974. Distribución Superficial de Algunos Parámetros Hidrológicos Físicos y Quínicos, en el Estero de Punta Banda, B.C. en Otoño e Invierno. Ciencias Marinas, Vol. 1, No. 1: 16-45.

Alvarez Borrego S.,G. Ballesteros Gri.jalva, y A. Chee Ba.. rragán. 1975. Hidrología de la Bahía de San Quintin, Baja California en Verano, Utorio e Invierno. Ciencias Marinas, Vol. 2, No.2:1-9.

Alvarez Borrego S. y A. Chee Barragán. 1976. Distribución Superficial de Fosfatos y Silicatos en Bahía San Quintin, B.C. Ciencias Marinas, Vol. 3, No. 1: $5 l-6 I$. 
Alvarez Bcrrego S. y C. López Alvarez. 1975. Distribución de Biomasa de Fitoplancton por Grupos Taxonómicos en Bahía San Quintín, B.C. a través de un Ciclo Anual. Reporte para I.N.P. de la S.I.C. y la Dirección de Acuacultura de la S.R.H. (No publicado).

Cabrera Muro, H. 1972. Distribución de Temperatura en la Bahía de Todos Santos, (junio-octubre). Ciencias Marinas, Vol. 1, No. 1: 65-77.

Contreras Rivas, I. 1973. Influencia Termohalina de las aguas del Estero de Punta Banda en la Bahía de Todos Santos, B.C. Tésis profesional. Escuela Superior de Ciencias Marinas, Universidad Autonoma de Baja California.

Chávez de Nishikawa A. G. y S. Alvarez Borrego. 1974. Hidrología de la Bahía de San Quintín, Baja California en Invierno y Primavera. Ciencias Marinas, Vol. 1 No. 2: 31-62.

Chávez de Ochoa, C. 1975. Algunas Condiciones de Surgencias Durante la Primavera de 1974, para el Area Adyacente a Punta Banda, Baja California. Ciencias Marinas, Vol. 2, No. 2: 111-124.

Dawson E. Y. 1951. A Further Study of Upwelling and Vegetation along Pacific Baja California, Mexico. Jour. Mar. Res. Vol. 10, No. 1: 39-58.

Lara Lara J. R. y S. Alvarez Borrego. 1975. Ciclo Anual de Clorofilas y Producción Orgánica Primaria en Bahía San Quintín, B.C. Ciencias Marinas, Vol. 2, No. 1: 77-97.

Redfield A. C., B. H. Ketchum y F. A. Richards. 1963. The Influence of Organisms on the Composition of the Sea Water. En: The Sea: Ideas and Obervations on Progress in the Study of the Sea, M. N. Hill (Editor), Interscience, New York. Vol. 2, Cap.: 26-77.

Strickland, H. D. y T. R. Parson. 1965. A Practical Handbook of Sea Water Analysis, Fish. Res. Board of Canada, Bull. 167. 311 p.p. 